\title{
Alcoholysis-Assisted Exfoliation of Boron Nitride Nanosheets from Hexagonal Boron Nitride
}

\author{
Witita Laosamathikul, Toshiki Sawada and Takeshi Serizawa* \\ Department of Chemical Science and Engineering, Tokyo Institute of Technology, \\ 2-12-1 Ookayama, Meguro, Tokyo 152-8550, Japan \\ * Corresponding author: serizawa@polymer.titech.ac.jp
}

\begin{abstract}
Boron nitride nanosheets (BNNSs) are attractive 2-dimensional nanomaterials with excellent thermal conductivity, mechanical properties, and thermal stability. It is therefore significant to develop new simple methods to produce BNNSs. In this study, BNNSs with nanometer thicknesses and diameters of several hundreds of nanometers were produced by alcoholysis-assisted exfoliation from hexagonal boron nitride (h-BN) through sonication of h-BN in primary alcohols such as butanol and hexanol. Atomic force microscopy images of the BNNSs revealed that the morphologies were dependent on alcohol species and preparation methods. Infrared absorption spectra of the BNNSs suggested that alcoholysis of B-N bonds in primary alcohols during sonication processes was essential for BNNS production.

Key words: boron nitride nanosheet, hexagonal boron nitride, alcoholysis, exfoliation
\end{abstract}

\section{INTRODUCTION}

Boron nitride $(\mathrm{BN})$ has an analogous structure of carbon. Three different structures are known for $\mathrm{BN}$; layered (so-called hexagonal BN (h-BN)), cubic, and tubular structures, ${ }^{1}$ which are analogs of graphite, diamond, and carbon nanotubes, respectively. $\mathrm{BN}$ has unique physicochemical properties such as excellent thermal conductivity, mechanical stiffness, and thermal stability. ${ }^{2}$ Therefore, BN was potentially used for various applications such as transistors, energy storages, thermal conductors, and topological insulators. ${ }^{3}$ Recently, boron nitride nanosheets (BNNSs) with a single or few layers of $\mathrm{h}-\mathrm{BN}$ have received much attention as relatively new 2-dimensional nanomaterials. $^{4}$ BNNSs can be produced by sonication-assisted exfoliation from h-BN in suitable solvents, ${ }^{5}$ chemical blowing of precursor polymers, ${ }^{6,7}$ plasma etching of BN nanotubes (BNNTs), ${ }^{7,8}$ and microwave plasma chemical vapor deposition of precursor gases on silicon substrates. ${ }^{9}$ However, it is still significant to develop new simple preparation methods of BNNSs.

We have previously demonstrated the chemical peeling of BNNTs through sonication-assisted alcoholysis of BNNTs in primary alcohols under ambient conditions, followed by partial production of BN nanoribbons. ${ }^{10}$ Morphological analysis and DFT calculation suggested that the reactions more readily occurred in alcohols with longer alkyl chains. We hypothesized from this previous study that the reactions could be extended to h-BN to simply produce BNNSs. In this study, BNNSs were produced by alcoholysis-assisted exfoliation from h-BN through sonication of h-BN in primary alcohols (Fig. 1). Butanol and hexanol, which showed great reactivities with BNNTs, ${ }^{10}$ were used for reactants with h-BN as well as solvents for the obtained BNNSs. The effect of pre-treatment of h-BN by mechanical grinding on the production of BNNSs was also analyzed. The morphologies and chemical structures of BNNSs were characterized by atomic force microscopy (AFM) and Fourier-transform infrared (FT-IR) absorption spectroscopy, respectively.

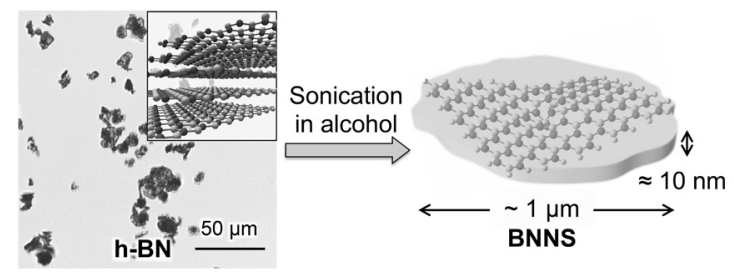

Fig. 1 Schematic representation of this study.

\section{EXPERIMENTAL SECTION}

2.1 Preparation of BNNSs from h-BN

Two methods to exfoliate BNNSs from h-BN were performed. For Method 1, $3 \mathrm{~mL}$ of butanol (nacalai tesque) or hexanol (Sigma-Aldrich) was added to $10 \mathrm{mg}$ of h-BN (Sigma-Aldrich, $\sim 1 \mu \mathrm{m}$ in size) in a glass tube, and then the mixtures were sonicated in a Branson Advanced Sonifier (Model 250 AA, output power at 30 W) with a cooling system using tap water (approximately $15{ }^{\circ} \mathrm{C}$ ) for $3 \mathrm{~h}$, followed by centrifugation at $2000 \mathrm{rpm}$ for $25 \mathrm{~min}$ to remove remained h-BN. After that, the products in supernatants were characterized. For Method 2, $0.1 \mathrm{~mL}$ of butanol or hexanol was added to $10 \mathrm{mg}$ of h-BN in a mortar, and then the mixtures were manually ground for $30 \mathrm{~min}$ at ambient temperature. It is noted that alcohols were re-added several times into the mixtures during grinding to make the volume of alcohols constant as much as possible. After that, the volume of alcohols was adjusted to $3 \mathrm{~mL}$, and then the mixtures were further ground for 5 min. Finally, the mixtures were sonicated and centrifuged similarly to Method 1, followed by 
characterization of the products in supernatants. To estimate the percent yields of the products in supernatants, the product amounts were estimated by subtracting the amounts of precipitates after centrifugation from the initial amounts of h-BN. Alcohol solvents for the precipitates were dried in an oven at $110{ }^{\circ} \mathrm{C}$ for $24 \mathrm{~h}$. The percent yields were estimated from experiments in triplicate.

\subsection{Characterization of BNNSs}

For AFM observations, adequate amounts of supernatants were spin-cast on a freshly cleaved mica substrate at $600 \mathrm{rpm}$, followed by drying at ambient temperature. Images were obtained with an SPM-9600 microscope (Shimadzu) in a tapping mode using standard silicon cantilevers under ambient conditions. The thicknesses of BNNSs were estimated from cross-sectional images. The diameters of BNNSs were estimated by averaging the long- and short-axes of BNNSs.

For FT-IR absorption spectra, adequate amounts of supernatants were cast on a gold-coated poly(ethylene terephthalate) film. The substrate was set on FT/IR-4100 (JASCO) with attenuated total reflection attachments. Spectra were obtained with the cumulative number of 100 under ambient conditions.

\section{RESULTS AND DISCUSSION}

The colorless and transparent supernatants that contained the products were obtained under all experimental conditions. The percent yields of the products in supernatants were estimated to be approximately $30 \%$ under all experimental conditions, suggesting that alcohol species and preparation methods did not affect the total amounts of the products exfoliated from h-BN.

Fig. 2 shows the morphological analyses of the products obtained by Method 1, in which the mechanical grinding was not performed before sonication. Significantly, round-shaped nanosheet-like objects were observed from the AFM images, irrespective of alcohol species, suggesting that the exfoliation of BNNSs from h-BN were successfully demonstrated by sonication treatment of h-BN in primary alcohols. The thickness and its distribution of the products obtained using hexanol were smaller than those obtained using butanol. The mean thickness for hexanol was estimated to be approximately $3 \mathrm{~nm}$, which corresponded to a 6-layer structure of h-BN. ${ }^{11}$ Furthermore, the distribution of diameters for hexanol was also smaller than that for butanol. The mean diameter for hexanol was estimated to be approximately $130 \mathrm{~nm}$. It was therefore suggested that hexanol was a more effective reactant to produce BNNSs with more homogeneous morphologies by Method 1. Our previous study demonstrated that sonication-assisted alcoholysis of $\mathrm{B}-\mathrm{N}$ bonds more readily occurred in primary alcohols with longer alkyl chains. ${ }^{10}$ A possible reason for the greater reactivities is derived from the greater electron densities of hydroxyl groups for primary alcohols with longer alkyl chains due to hyperconjugation of alkyl chains. Therefore, the greater reactivity of hexanol might promote the production of morphologically homogeneous BNNSs from h-BN. (a)
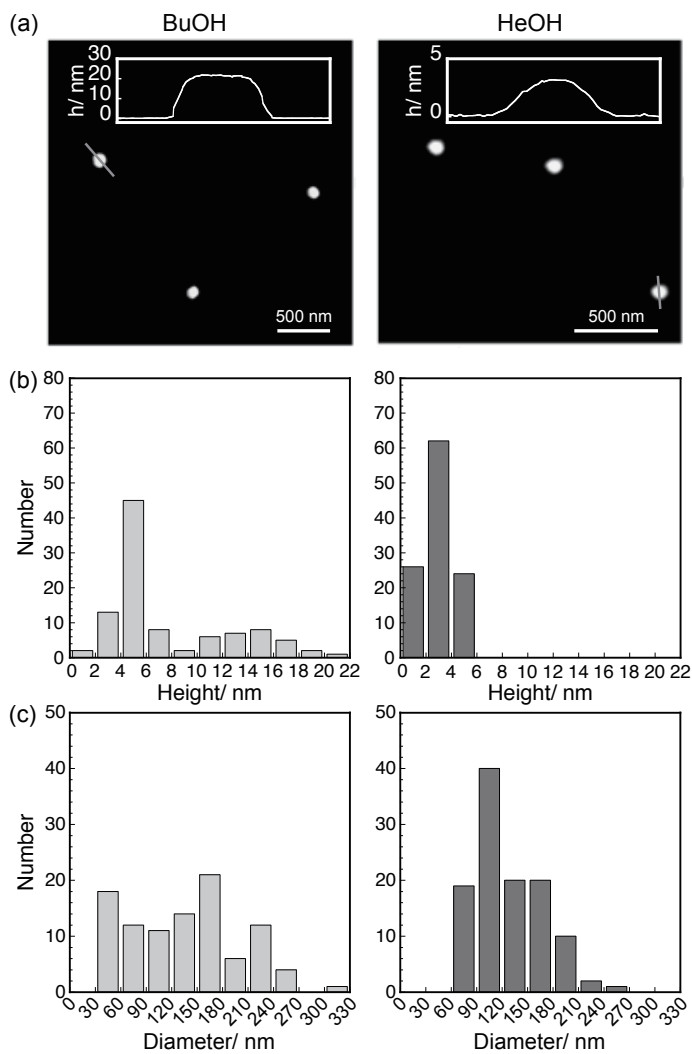

Fig. 2 Morphological analyses of the products obtained in butanol (left) and hexanol (right) by Method 1. (a) AFM images, (b) histograms for thickness, and (c) histograms for diameters.

In order to evaluate the effect of h-BN pre-treatment on the production of BNNSs, mechanical grinding of h-BN was performed before sonication as Method 2. Fig. 3 shows the photos of the mixtures composed of h-BN and alcohols with or without grinding. With grinding, the greater amounts of h-BN were dispersed in alcohols even after standing for $3 \mathrm{~h}$, suggesting that the size of h-BN became small through grinding. Fig. 4 shows the AFM images and morphological analyses of the products. The thickness of the products obtained in both alcohols tended to decrease, while the diameter tended to increase, compared with the products obtained by Method 1 (see Fig. 2). It is noted that the distributions of

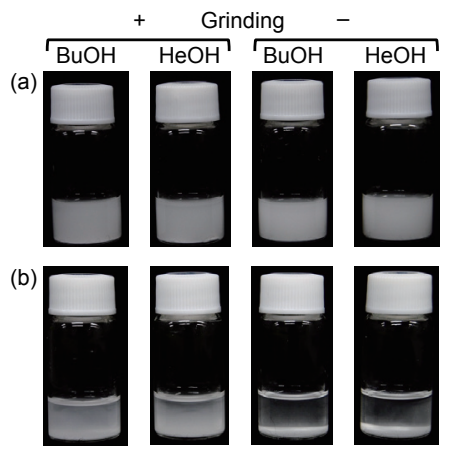

Fig. 3 Photos of the mixtures composed of h-BN and alcohols before and after grinding. Photos were taken (a) just after grinding and (b) after standing for $3 \mathrm{~h}$. 
(a)

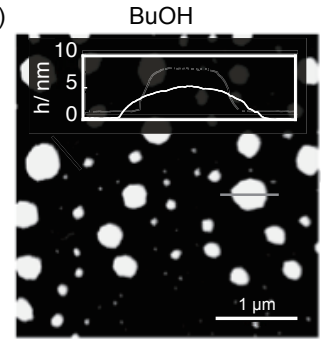

(b)
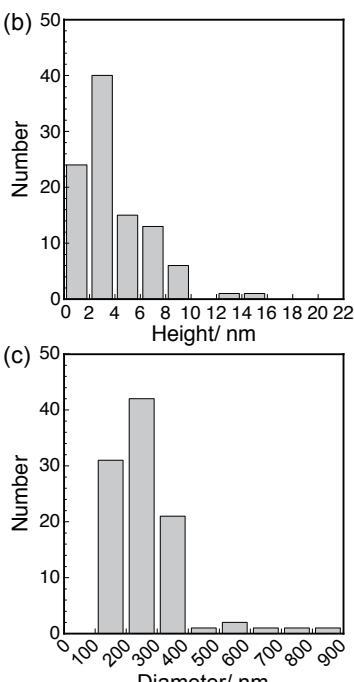

Diameter/ nm
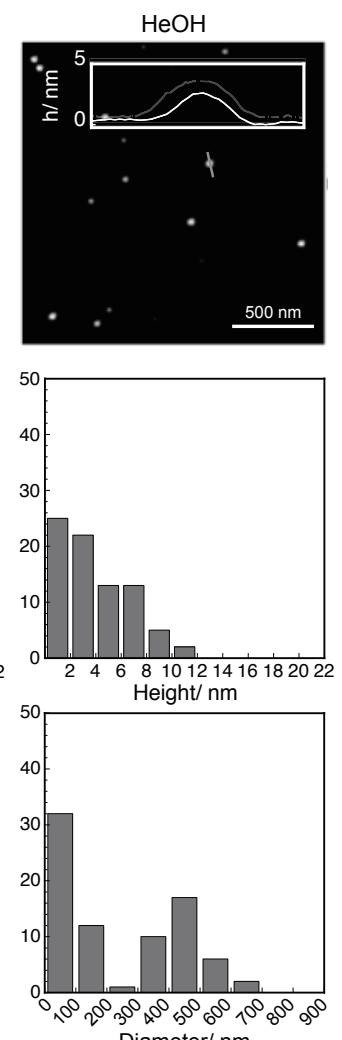

Fig. 4 Morphological analyses of the products obtained in butanol (left) and hexanol (right) by Method 2. (a) AFM images, (b) histograms for thickness, and (c) histograms for diameters.

thickness and diameter for hexanol became large, compared with the products obtained by Method 1. The mean thickness and diameter for butanol were estimated to be approximately $4 \mathrm{~nm}$ and $260 \mathrm{~nm}$, respectively. It was therefore found that mechanical pre-treatment of h-BN has potentials for controlling the morphologies of BNNSs exfoliated by sonication processes.

The chemical structures of the products were characterized by FT-IR absorption spectroscopy. Fig. 5 shows the spectra for all the products. In all spectra, two absorption peaks were observed at around 1260 and 723 $\mathrm{cm}^{-1}$, which can be assigned to $\mathrm{B}-\mathrm{N}$ stretching vibration and $\mathrm{B}-\mathrm{N}-\mathrm{B}$ bending vibration bands of $\mathrm{h}-\mathrm{BN}$, respectively. ${ }^{12}$ Absorption peaks were also observed at around $1720,1105,1021$, and $510 \mathrm{~cm}^{-1}$. These peaks can be assigned to $\mathrm{B}-\mathrm{O}$ stretching, $\mathrm{C}-\mathrm{O}$ stretching, $\mathrm{B}-\mathrm{O}-\mathrm{C}$ stretching, and $\mathrm{O}-\mathrm{B}-\mathrm{O}$ bending vibration bands, respectively. ${ }^{11,13-16}$ Absorptions in the region of $2920-2850 \mathrm{~cm}^{-1}$ can be assigned to $\mathrm{C}-\mathrm{H}$ stretching vibration bands. These peaks were similarly observed for the chemical peeling of BNNTs through sonication-assisted alcoholysis of BNNTs in primary alcohols. ${ }^{10}$ All these observations strongly suggested that the production of BNNSs was originated from alcoholysis of B-N bonds of h-BN in primary alcohols during sonication processes. It was therefore found that sonication-assisted alcoholysis proceeded similarly for h-BN with a flake structure, which was different from BNNTs. However, since the obtained BNNSs had

round-shape, directional unzipping for the production of BNNRs from BNNTs ${ }^{10}$ appeared to hardly proceed for h-BN. There must be many alkyl groups derived from primary alcohols at least at the edge of BNNSs. More detailed structures of BNNSs will be analyzed in the near future.

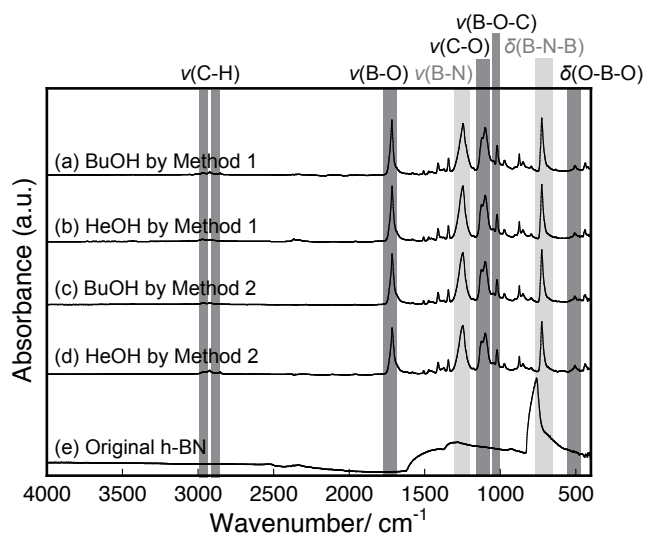

Fig. 5 FT-IR absorption spectra of the products obtained in (a) butanol and (b) hexanol by Method 1, the products obtained in (c) butanol and (d) hexanol by Method 2, and (e) original h-BN.

\section{CONCLUSION}

Sonication of h-BN, which was pre-treated with or without mechanical grinding, in butanol and hexanol exfoliated BNNSs with nanometer thicknesses and diameters of several hundreds of nanometers. The percent yields of BNNSs were estimated to be approximately $30 \%$, which were irrespective of alcohol species and preparation methods. AFM revealed that the morphologies of BNNSs were dependent on alcohol species and preparation methods. FT-IR absorption spectroscopy showed the representative peaks assigned to the chemical bonds for alcoholysis of $\mathrm{B}-\mathrm{N}$ bonds. The present sonication-assisted alcoholysis of h-BN will be potentially useful for simple production of functional BNNSs.

\section{REFERENCES}

[1] R. T. Paine; C. K. Narula, Chem. Rev., 90, 73. (1990).

[2] W. Schnick, Angew. Chem., Int. Ed., 32, 806. (1993).

[3] K. Watanabe; T. Taniguchi; H. Kanda, Nat. Mater., 3, 404. (2004).

[4] Y. Lin; J. W. Connell, Nanoscale, 4, 6908. (2012).

[5] C. Y. Zhi; Y. Bando; C. Tang; H. Kuwahara; D. Golberg, Adv. Mater., 21, 2889. (2009).

[6] X. Wang; C. Zhi; L. Li; H. Zeng; C. Li; M. Mitome; D. Golberg; Y. Bando, Adv. Mater., 23, 4072. (2011).

[7] X. Wang; A. Pakdel; C. Zhi; K. Watanabe; T. Sekiguchi; D. Golberg; Y. Bando, J. Phys.: Condens. Matter, 24, 314205. (2012).

[8] H. Zeng; C. Y. Zhi; Z. Zhang; X. Wei; X. Wang; W. Guo; Y. Bando; D. Golberg, Nano Lett., 10, 5049. (2010).

[9] C. Zhang; X. Hao; Y. Wu; M. Du, Mater. Res. Bull., 47, 2277. (2012). 
[10] D. Kim; S. Nakajima; T. Sawada; M. Iwasaki; S. Kawauchi; C. Y. Zhi; Y. Bando; D. Golberg; T. Serizawa, Chem. Commun., 51, 7104. (2015).

[11] A. Pakdel; Y. Bando; D. Golberg, Chem. Soc. Rev., 43, 934. (2014).

[12] L. Ansaloni; E. de Sousa, Mater. Sci. Appl., 4, 22. (2013).

[13] E. I. Kamintsos; M. A. Karakassides; G. D. Chryssikos, J. Phys. Chem., 91, 1073. (1987).

[14] R. E. Zeebe, Geochim. Cosmochim. Acta, 69, 2753. (2005).

[15] I. Yanase; R. Ogawara; H. Kobayashi, Mater. Lett., 63, 91. (2009).

[16] C. Y. Zhi; N. Hanagata; Y. Bando; D. Golberg, Chem. Asian J., 6, 2530. (2011).

(Received July 26, 2017; Accepted September 3, 2017;

Published Online October 1, 2017) 